\section{AML: Langzeitprognose schlechter als vermutet}

In der Vergangenheit gab es Hinweise, dass Patienten mit akuter myeloischer Leukämie (AML), die 2 Jahre nach autologer Stammzelltransplantation (ASCT) rezidivfrei sind, eine sehr gute Prognose haben. Dies scheint zu optimistisch.

$T^{\mathrm{s}}$ omasz Czerw und Kollegen überprüften retrospektiv anhand des Registers der European Society for Blood and Marrow Transplantation (EBMT) den Langzeiterfolg der Transplantation nach 5 und 10 Jahren. Die Befunde von 3.567 AML-Patienten, die zwischen 1990 und 2008 eine ASCT in der 1. oder 2. Remission erhalten hatten, konnten ausgewertet werden. Im Median waren die Betroffenen 45 Jahre alt.

Mit einem Anteil von 17,3\%, 26,6\% und $22,4 \%$ waren die meisten Patienten den FAB-Klassen M1 (AML ohne Ausreifung), M2 (AML mit Ausreifung) und M4 (akute myelomonozytäre Leukämie) zuzuordnen. Bei $32 \%$ der Patienten waren die autologen Stammzellen aus dem Knochenmark isoliert worden, bei $68 \%$ stammten sie aus dem peripheren Blut.
Die Konditionierung wurde bei 74,4\% der Patienten mittels Chemotherapie vorgenommen, bei 21,1\% mittels Ganzkörperbestrahlung.

Median betrug das Follow-up 6,9 Jahre. Die Wahrscheinlichkeit, 5 bzw. 10 Jahre nach der Transplantation leukämiefrei zu leben, betrug 86 bzw. 76\%. Nach 5 Jahren lag die kumulative Rezidiv-Inzidenz bei $11 \%$ und nach 10 Jahren bei $16 \%$. Das bedeutet nach Ansicht von Czerw und Kollegen, dass die früheren Annahmen einer guten Prognose falsch sind. Schließlich berechneten die Onkologen für die kumulative, nicht durch Rezidive bedingte Mortalität nach 5 bzw. 10 Jahren einen Wert von 3 bzw. $8 \%$.

Negativ wirkten sich auf das leukämiefreie Überleben außer hohem Alter auch die Verwendung von peripheren Stammzellen sowie die FAB-Stadien M0, M6 und M7 aus, wie aus der multivariaten Analyse hervorging. Alle 3 Parameter bestimmten auch das Rezidivrisiko. Möglicherweise sei das Risiko der Übertragung von Leukämiezellen bei der Verwendung autologer Stammzellen aus peripherem Blut größer als bei Knochenmark, vermuten die Onkologen. Sie schlagen vor, künftig bei Gewinnung der Stammzellen die minimale Resterkrankung zu bestimmen.

Fazit: Patienten mit AML, die eine ASCT erhalten, haben langfristig eine schlechtere Prognose als vermutet. Vor allem wirkt sich offenbar die Verwendung von Stammzellen aus dem peripheren Blut negativ aus.

Peter Leiner

Czerw T et al. Long-term follow-up of patients with acute myeloid leukemia surviving and free of disease recurrence for at least 2 years after autologous stem cell transplantation: A report from the acute leukemia working party of the European Society for Blood and Marrow Transplantation. Cancer. 2016 Mar 28. [Epub ahead of print].

\section{Unabhängiger Prognosefaktor MRD bei AML}

\author{
Bei AML-Patienten mit Standardrisiko ist unklar, ob sie eher von einer \\ Chemotherapie oder einer Stammzelltransplantation profitieren. Britische \\ Onkologen haben deshalb geprüft, ob Hinweise auf eine minimale Rest- \\ erkrankung (MRD) prognostische Informationen liefern können.
}

W ird bei Patienten mit akuter lymphatischer Leukämie (ALL) eine MRD nachgewiesen - sei es am Ende der Induktionstherapie oder der Konsolidierung oder vor einer allogenen Stammzelltransplantation -, dann ist die Prognose signifikant schlechter als bei fehlendem MRD-Nachweis. Daran erinnert Michael J. Burke in einem Kommentar zur folgenden Studie.

Um das Rezidivrisiko nach Therapie auch bei akuter myeloischer Leukämie (AML) besser abschätzen zu können, analysierten Adam Ivey und Kollegen die Daten von Patienten der britischen AML17-Studie. Die Patienten hatten ein mutiertes NPM1-Gen, das bei jedem dritten Patienten mit zytogenetischem Standardrisiko nachweisbar ist. In dieser
Gruppe ist die Bedeutung der Stammzelltransplantation bisher unklar. Die Onkologen analysierten peripheres Blut von 346 Patienten mit NPM1-Mutation. Nach 2 Zyklen einer Induktionschemotherapie war bei $15 \%$ von ihnen die Mutation noch immer nachweisbar.

Dieser Nachweis einer MRD war verglichen mit Patienten ohne die Mutation mit einem erhöhten Rezidivrisiko 3 Jahre später assoziiert (82 vs. $30 \%$ ). Das Rezidivrisiko war fast um das 5-fache erhöht (Hazard Ratio [HR] 4,80; $95 \%$-Konfidenzintervall [95\%-KI] 2,957,90; $\mathrm{p}<0,001)$.

Außerdem war die Überlebenswahrscheinlichkeit signifikant verringert (24 vs. $75 \%$; HR für Tod 4,38, $95 \%$-KI 2,577,47; $\mathrm{p}<0,001)$. In der multivariaten
Analyse war der Nachweis der MRD der einzige unabhängige Prognosefaktor für Mortalität (HR 4,84, 95 \%-KI 2,57-9,15; $\mathrm{p}<0,001)$. Klinisch relevant ist das, weil AML-Patienten mit NPM1-Mutation eine der größten Subgruppen ausmachen. Sie galten bisher als Patienten mit Standardrisiko und der Nutzen einer Transplantation oder Chemotherapie allein war bei ihnen unklar. Als Hochrisikopatienten könnten sie von einer Transplantation eher profitieren als von einer Chemotherapie.

Fazit: Bei AML-Patienten mit NPM1Mutation liefert die MRD-Bestimmung wertvolle prognostische Informationen, die für eine verbesserte Therapiestrategie genutzt werden können - für Patienten, bei denen der Nutzen von Transplantation oder Chemotherapie bisher nicht klar war.

Peter Leiner

Ivey $A$ et al. Assessment of Minimal Residual Disease in Standard-Risk AML. N Engl J Med. 2016;374(5):422-33. - Comment: Burke MJ. Minimal Residual Disease in NPM1-Mutated AML. N Engl J Med. 2016;374(5):481-2. 